\title{
AOTrauma-Symposium „Alterstraumatologie“ am 17. November 2017 in Worms
}

Jochen Blum

Am 17. November 2017 fand im Herrnsheimer Schloss in Worms das AOTraumaSymposium „Alterstraumatologie - Osteosynthesen bei Frakturen des älteren Menschen" statt.

Kolleginnen und Kollegen aus der Unfallchirurgie beschäftigten sich mit neuen Entwicklungen im Bereich der Versorgung älterer Patienten nach Knochenbrüchen. Die wissenschaftlichen Leiter des AO-Symposiums, Prof. Dr. Jochen Blum vom Klinikum Worms und Prof. Dr. Paul A. Grützner von der BG-Klinik Ludwigshafen, organisieren diese Tagung in Kooperation bei jährlichen Themen- und Ortswechsel zwischen den Standorten Worms und Ludwigshafen.

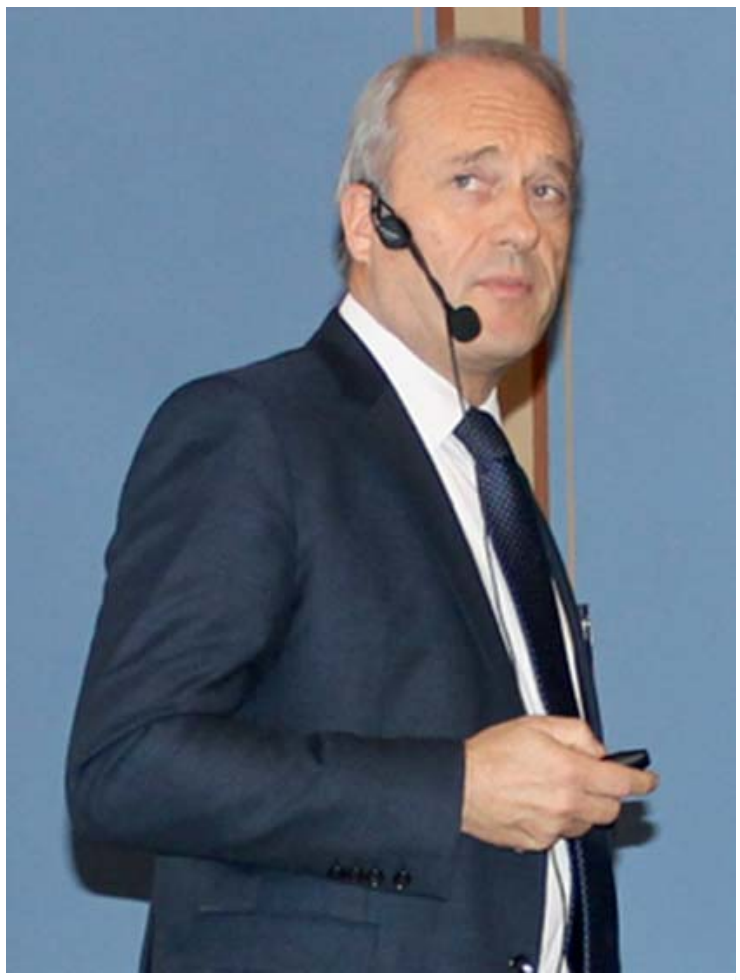

Abb. 1 Der wissenschaftlichen Leiter des AO-Symposiums, Prof. Dr. Jochen Blum bei der Begrüßung der Teilnehmer und Einführung in das Thema „Alterstraumatologie“. (๖) Prof. Jochen Blum
Besonderheit in diesem Jahr war, dass neben den unfallchirurgischen Fachleuten auch 2 Chefärztinnen aus dem Bereich der Geriatrie zu Vorträgen und Diskussion geladen waren. Die Chefärztin der Geriatrie am Klinikum Worms, Frau Dr. Buchwald, berichtete sowohl, was die Geriatrie für die betagten unfallchirurgischen Patienten nach der operativen Phase beitragen kann, als auch was sie von den Unfallchirurgen an kooperativen Abläufen verlangt, um die gemeinsame Behandlung in Bereich der Alterstraumatologie zu optimieren. Die zunehmende Steigerung unserer Lebenserwartung erfordert neue

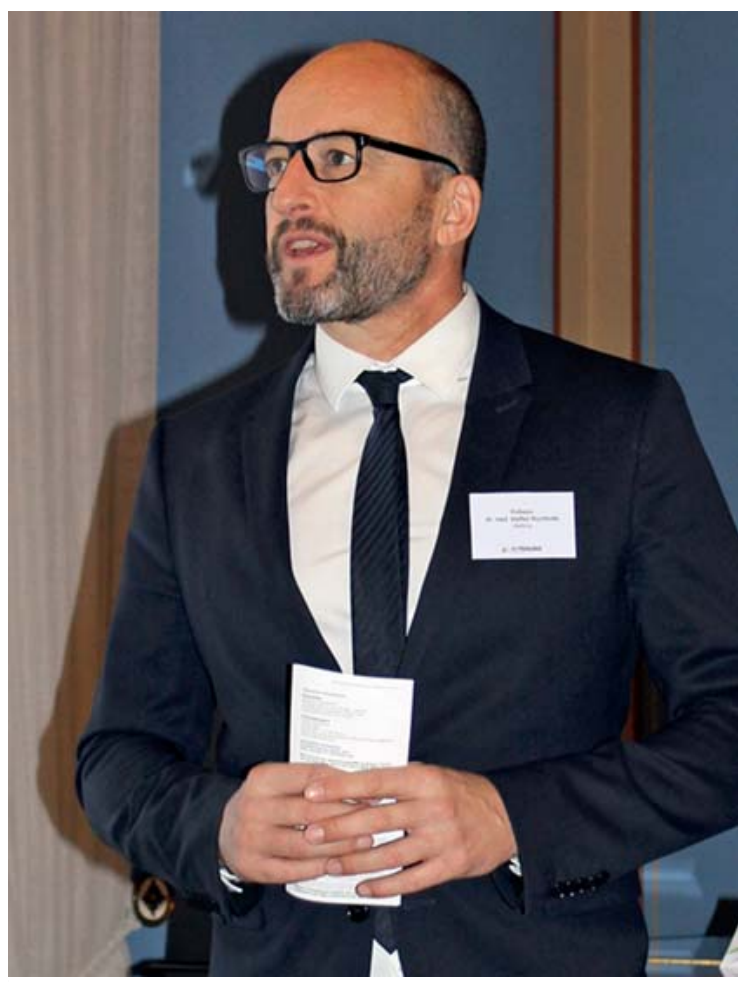

- Abb. 2 Prof. Dr. med. Steffen Ruchholtz erläutert die aktuellen Konzepte zu Schenkelhalsfrakturen und proximalen Femurfrakturen im hohen Alter. @ Prof. Jochen Blum 


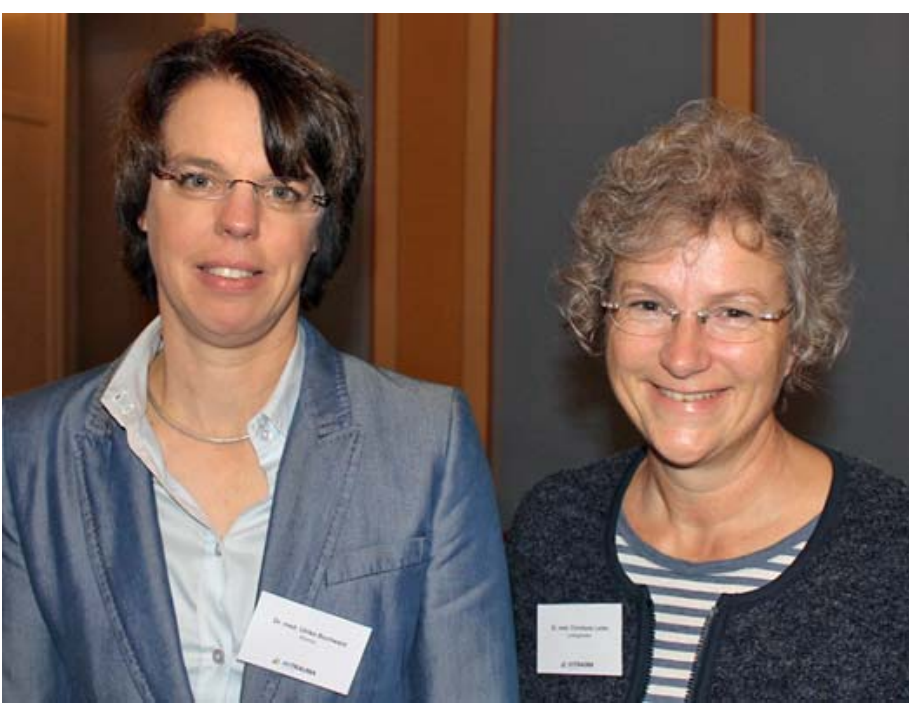

- Abb. 3 Die beiden Chefärztinnen aus dem Bereich der Geriatrie, Frau Dr. Buchwald (Klinikum Worms) und Frau Dr. Lenke (BG-Unfallklinik Ludwigshafen). (๑ Prof. Jochen Blum

Konzepte in der medizinischen wie auch sozialen Betreuung unserer älteren Mitbürger und Mitbürgerinnen, die vor einigen Jahren noch nicht derart im Mittelpunkt standen.

Vonseiten der AO wurden einerseits neue chirurgische Implantate und Operationstechniken entwickelt, aber andererseits wurde die altersgerechte medizinische Betreuung dieser wachsenden Patientengruppe verbessert, die deren Begleiterkrankungen, reduzierte Mobilität, aber auch deren soziale Besonderheiten berücksichtigt, wo ältere Menschen zunehmend entweder alleine zu Hause leben, oft auch einen pflegebedürftigen Ehepartner mitbetreuen, oder auch nähere Angehörige weit weg woh- nen. Frau Dr. med. Christiane Lenke, Geriatrische Leiterin der Alterstraumatologie der BG-Unfallklinik Ludwigshafen, stellte das dortige Konzept „LUISA - Ludwigshafener interdisziplinäres Zentrum für Alterstraumatologie“ vor. Dies ergänzte das klassische AO-Symposiums-Konzept wissenschaftlicher Vorträge, Diskussionsrunden und praktischer Workshops im Bereich der Osteosynthese in besonderer Weise und die Gespräche zwischen Traumatologen und Geriatern waren entsprechend lebhaft.

Im Mittelpunkt der Workshops standen die zementaugmentierten geriatrischen Implantate, wie PHILOS und PFN-A, die Vorträge stellten je nach Körperregion die Besonderheiten altersbezogener Verletzungsarten wie auch Versorgungstechniken heraus. Gerade auch die neuen Beckenklassifikationen unter alterstraumatologischen Gesichtspunkten, präsentiert durch Prof. Dr. Dr. Pol Rommens, fanden besonderes Interesse.

Die wissenschaftlichen Leiter und Referenten waren von der hohen Beteiligung wie auch vom hohen Niveau der wissenschaftlichen Vorträge und Diskussionen begeistert. Die Resonanz der Teilnehmer waren sehr gut, allerdings wurde doch vermehrt die Bitte geäußert, die Diskussionszeit noch großzügiger einzuplanen, was im nächsten Jahr sicher gerne Berücksichtigung finden wird, denn die AO-Symposien leben vom offenen Gespräch und Austausch.

Prof. Jochen Blum, Worms

E-Mail: jochen.blum@klinikum-worms.de

Bibliografie

DOI https://doi.org/10.1055/a-0591-2892

OP-JOURNAL 2018; 34: 77-78 ๔ Georg Thieme Verlag KG Stuttgart · New York ISSN 0178-1715 